\title{
Evaluación técnica de un sistema tradicional de cosecha en plantaciones de Eucalyptus globulus de corta rotación en Valdivia, Chile
}

\author{
Technical evaluation of a traditional harvesting system in short rotation \\ age of Eucalyptus globulus stands in Valdivia, Chile
}

\author{
Patricio Carey Briones ${ }^{1 *}$, Alberto Figueroa Sotomayor ${ }^{2}$, Patricio Valenzuela Cavieres ${ }^{2}$ \\ *Autor de Correspondencia: ${ }^{1}$ Universidad Austral de Chile, Instituto de Manejo Forestal, Casilla 567, \\ Valdivia, Chile, pcarey@uach.cl \\ ${ }^{2}$ Universidad Austral de Chile, Ingeniero Forestal, Casilla 567, Valdivia, Chile.
}

\begin{abstract}
SUMMARY
A traditional harvesting system, with hand fell and oxen logging was technically evaluated in a clear-cut of Eucalyptus globulus. The study area is a short rotation age plantation stand with $0.15 \mathrm{~m}^{3} /$ tree located in Valdivia, south of Chile. The activities were carried out by a crew of ten forest workers using chainsaw and hand-tools, who executed simultaneously six subsystems (felling, delimbing, logging, bucking, debarking and stacking). Felling and bucking were carried out by means of a chainsaw Sthil 017; the production rates observed were 7.1 and $9.2 \mathrm{~m}^{3} / \mathrm{h}$ respectively. Delimbing, debarking and stacking were executed with hand tools, and the production rate observed was $20.9 \mathrm{~m}^{3} / \mathrm{h}$ in delimbing and 8.3 and $10.3 \mathrm{~m}^{3} / \mathrm{h}$ in debarking and stacking. In the logging phase two yokes of oxen were used, the average logging distance was $50 \mathrm{~m}$ in favourable slopes conditions. A production rate of $1.9 \mathrm{~m}^{3} / \mathrm{h}$ per yoke of oxen was observed, this low rate was a consequence of dense underbrush in the study area. The study also considers a non-productive time (delays) analysis. A high frequency of delays because of resting times was observed. This is a characteristic of a labour intensive system based on the use of hand tools. These delays can be minimized by improving the working method and applying a training program.
\end{abstract}

Key words: oxen logging, harvesting productivity, hand-tools harvesting.

\section{RESUMEN}

En este estudio se determina el rendimiento $\left(\mathrm{m}^{3} / \mathrm{h}\right)$ de un sistema tradicional de cosecha a tala rasa de una plantación de Eucalyptus globulus, de corta rotación, ubicada en la provincia de Valdivia, zona centro-sur de Chile. Mediante un estudio de tiempos detallado, se determina el porcentaje de productividad de las actividades de cosecha y se determinan los tiempos ocasionales no productivos (demoras) que inciden en la productividad del sistema. Las actividades de cosecha fueron realizadas por una cuadrilla de diez trabajadores forestales, los cuales ejecutaron en forma simultánea los seis subsistemas que corresponden a: volteo, desrame, extracción, trozado, descortezado y apilado. El volteo y trozado fue realizado mediante motosierra obteniéndose los mayores rendimientos individuales, con 7,1 y $9,2 \mathrm{~m}^{3} / \mathrm{h}$, respectivamente. Los subsistemas desrame, descortezado y apilado fueron ejecutados mediante herramientas manuales, como hachas y ganchos, presentando el mayor rendimiento el desrame con hacha, con $20,9 \mathrm{~m}^{3} / \mathrm{h}$. En descortezado y apilado, en cambio, presentaron rendimientos menores, logrando 8,3 y $10,3 \mathrm{~m}^{3 /}$ h. La extracción fue ejecutada por dos yuntas de bueyes, las que no obstante trabajar en condiciones de distancia y pendiente favorables, presentaron bajo rendimiento individual de $1,9 \mathrm{~m}^{3} / \mathrm{h} / \mathrm{hombre}$, como resultado de un denso sotobosque. El estudio considera además un registro y análisis de los tiempos no productivos, entre los cuales se destaca una alta ocurrencia de pausas por descanso de los trabajadores, característico de los sistemas basados en la utilización de herramientas manuales. Estas pausas pueden ser minimizadas mediante una mayor capacitación del personal y una mejor planificación y organización de las actividades.

Palabras clave: extracción con bueyes, productividad y rendimiento de cosecha, cosecha tala rasa eucaliptos.

\section{INTRODUCCIÓN}

De los sistemas de cosecha forestal, el que involucra una mayor ocupación de mano de obra es el sistema tradicional, mediante el uso de motosierras en el volteo y animales de tiro en la extracción (FAO 1995). Este siste- ma intensivo en la ocupación de mano de obra se aplica principalmente en bosques de pequeños propietarios forestales, donde no se tiene como objetivo una operación de gran escala, sino más bien producir un menor impacto ambiental con menores requerimientos de inversión. De esta forma, pequeños empresarios forestales ven en este 
sistema una solución rentable, ya que en todos los casos no se cuenta con la capacidad de solventar económicamente una faena mecanizada por la mayor inversión, gasto en reparaciones y manutención que implica (Otavo 1984).

Los sistemas tradicionales, con extracción mediante bueyes, son empleados a menudo por algunos empresarios forestales, ya sea en raleos o en faenas de cosecha a tala rasa, debido a la gran versatilidad, resistencia y fuerza de los bueyes, además del menor impacto generado tanto en el suelo como en la vegetación remanente (Dykstra y Heinrich 1996).

En Chile se han establecido más de 342.000 hectáreas de Eucalyptus sp. (INFOR 2002). Estas plantaciones están siendo cosechadas con edades 10 a 12 años para madera pulpable, las que se caracterizan por presentar árboles delgados (diámetros medios de 15 a $20 \mathrm{~cm}$ ), con alturas de 16 a $22 \mathrm{~m}$ y volúmenes por árbol medio a la edad de cosecha de 0,10 a $0,20 \mathrm{~m}^{3}$. Sin embargo, en el país existe poca documentación sobre este tipo de faenas en plantaciones de corta rotación, por lo cual este trabajo se plantea como objetivo generar información sobre rendimientos de un sistema tradicional de cosecha en plantaciones de E. globulus Labill de corta rotación en la Décima Región de Chile.

\section{MÉTODOS}

Zona de estudio. El área de estudio se ubica en el fundo Promesa a $22 \mathrm{~km}$ al suroeste de la ciudad de Valdivia, en la zona centro-sur de Chile y corresponde a una plantación de E. globulus de 10 años, con 1.504 árboles/ha y un volumen medio por árbol de $0,15 \mathrm{~m}^{3}$. El sotobosque es denso y dificulta el desplazamiento, y está compuesto por quila (Chusquea quila Kunth). La zona presenta un clima templado lluvioso, con precipitación media anual de $2.351 \mathrm{~mm}$. La topografía del entorno se caracteriza por lomajes suaves, con pendientes que no superan el $25 \%$.

Descripción del sistema de cosecha. El sistema de cosecha en estudio presentó alta ocupación en mano de obra y estuvo compuesto de la siguiente secuencia de actividades o subsistemas:

- Volteo: realizado por un operador en forma manual, mediante motosierra de 1,5 Hp (Stihl 017) y el ciclo de trabajo incluyó las etapas de selección de árbol, limpieza de vías de escape, despeje de base, corte de dirección y corte de caída.

- Desrame: a cargo de siete trabajadores forestales o "hacheros", los cuales realizaron manualmente el desrame y despunte de los árboles apeados. Para esto se emplearon hachas de mango recto de $0,7 \mathrm{~m}$.

- Extracción ("madereo"): ejecutado por dos yuntas de bueyes provistas de aperos y cadenas (figura 1). Los bueyes presentaron buenas condiciones sanitarias, con un peso promedio de $600 \mathrm{~kg}$ por ejemplar. La extracción se realizó principalmente a favor de la pendiente y con una distancia promedio de $50 \mathrm{~m}$.

- Trozado: actividad realizada por el mismo operador del volteo, mediante motosierra de 1,5 Hp (Stihl 017), quien una vez obtenido un stock de árboles suficiente para el desrame, se dirigió hasta la zona de acopio, a orilla de camino, para realizar el trozado de los árboles acanchados, de los cuales se obtuvieron trozos pulpables de 2,44 $\mathrm{m}$ de longitud. Una vez finalizado el trozado, el operador reinició el volteo en el interior del bosque.

- Descortezado: se realizó en forma manual por los mismos siete trabajadores forestales (hacheros) del desrame, quienes debieron acudir periódicamente a la zona de acopio para descortezar las trozas (figura 2), provistos de hachas y ganchos metálicos.

- Apilado: a continuación del descortezado, los trabajadores procedieron al apilado manual de los trozos. Esta actividad fue realizada a orilla de camino mediante ganchos metálicos, construyendo pilas de trozos de un metro de altura (metros ruma). Al concluir con el apilado, los trabajadores debieron dirigirse nuevamente a la zona de volteo para continuar con el desrame.

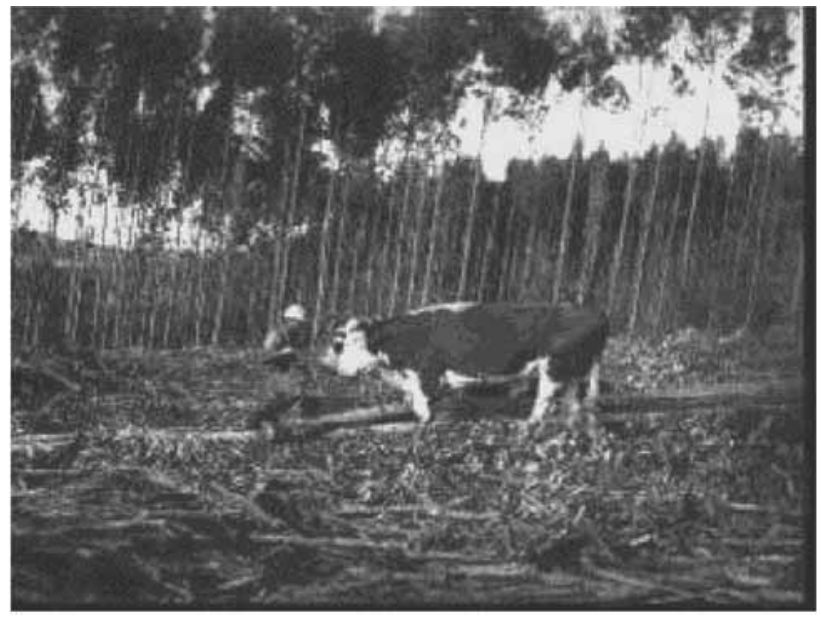

Figura 1. Extracción de trozas largas de Eucalyptus globulus en cosecha a tala rasa.

Oxen logging in tree-length system in Eucalyptus globulus clear-cut.

En consecuencia, el sistema de cosecha se compuso de una cuadrilla de 10 trabajadores forestales con herramientas manuales, más dos yuntas de bueyes. Excluyendo la actividad de extracción, todos los trabajadores de la cuadrilla debieron cumplir con más de una actividad durante el día, distribuyendo su jornada en al menos dos subsistemas.

Estudio de tiempo y productividad. Para la determinación de los volúmenes asociados a las diferentes activi- 


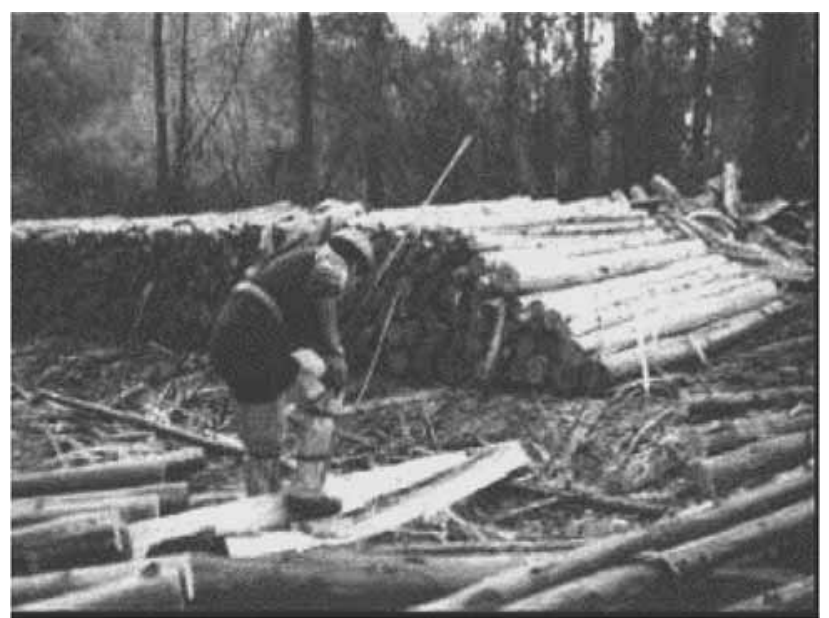

Figura 2. Descortezado manual a orilla de camino de trozas de Eucalyptus globulus.

Debarking with Hand-tools in pulp logs at roadside in Eucalyptus globulus clear-cut.

dades del sistema de cosecha se realizó un premuestreo, del cual se obtuvo el volumen medio por árbol y volumen medio por trozo. En los subsistemas volteo, desrame y extracción se empleó la ecuación 1 (ajuste propio) en el cálculo del volumen por árbol, donde $\mathrm{V}$ es volumen de madera ( $\left.\mathrm{m}^{3} / a ́ r b o l\right)$ hasta un índice de utilización de 8 $\mathrm{cm}$, DAP es el diámetro del árbol $(\mathrm{cm})$ a 1,3 m del suelo, y HT es la altura total (m):

$$
V=0,035+0,000023 \times D A P^{2} \times H T
$$

La cubicación en los subsistemas trozado, descortezado y apilado se determinó mediante la fórmula de Newton (Prodan et al. 1997) (ecuación 2), donde V es volumen $\left(\mathrm{m}^{3}\right) ; \mathrm{g}_{\mathrm{s}}, \mathrm{g}_{\mathrm{m}} \mathrm{y} \mathrm{g}_{\mathrm{i}}$ son el área basal del diámetro mayor, medio y menor $\left(\mathrm{m}^{3}\right)$, respectivamente; y $\mathrm{L}$ el largo de troza $(\mathrm{m})$ :

Cuadro 1. Resumen de tiempos y rendimientos por subsistemas. Summary of time study and production rates per subsystems.

$$
V=\left(g_{s}+4 g_{m}+g_{i}\right) \times L \div 6
$$

La metodología utilizada en la toma de tiempos correspondió al método de vuelta cero o tiempo de retorno (Currie 1979), diferenciando los siguientes conceptos descritos por Carey (1992):

- Tiempo planificado (hpl): tiempo total del ciclo de trabajo, incluyendo los tiempos de demora. Es decir, equivalió a la suma de tiempos productivos y demoras, expresándose en horas planificadas.

- Tiempo productivo (hpr): suma de los tiempos parciales de los elementos productivos del ciclo de trabajo de los subsistemas, excluyendo las demoras.

- Tiempo de demoras: tiempos ocasionales, ya sea indirectamente productivos o tiempos muertos. Se clasificó en tres grupos: operacionales, mecánicas y personales.

\section{RESULTADOS}

El menor porcentaje de productividad se registró en el volteo (cuadro 1) con tan sólo un 40,5\% de su tiempo total o planificado en labores productivas. La razón principal de esto fue el elevado tiempo de traslado hasta la zona de trozado por parte del operador, constituyendo la demora de mayor importancia para la actividad. Además, el sotobosque del lugar se caracterizó por la abundante presencia de quila, la que dificultó el desplazamiento. En consecuencia, los tiempos no productivos o demoras operacionales del volteo se componen de tiempos de desplazamiento hacia la zona de trozado (35\% de las demoras), tiempos de limpieza de la base del fuste $(19 \%$ de las demoras) y detenciones o pausas por sobre abastecimiento de madera (11\% de las demoras). Las demoras personales estuvieron compuestas exclusivamente por pausas de descanso del operador, con un $30 \%$ de las demoras (figura 3). Los restantes tiempos de demoras fueron de

\begin{tabular}{lcccccc} 
& Volteo & Desrame & Extracción & Trozado & Descortezado & Apilado \\
\hline $\mathrm{N}^{\circ}$ ciclos observados (n) & 690 & 174 & 339 & 155 & 526 & 524 \\
Tiempo total observado(hpl) & 14,5 & 8,6 & 61,4 & 21,1 & 9,8 & 7,9 \\
Tiempo productivo (hpr) & 5,9 & 4,4 & 43,4 & 10,8 & 7,1 & 6,0 \\
Tiempo no productivo (h) & 8,6 & 4,3 & 18,0 & 10,3 & 2,7 & 1,9 \\
Porcentaje de productividad (\%) & 40,5 & 50,4 & 70,7 & 51,4 & 72,3 & 76,4 \\
Duración actividad /jornada (hpl) & 4,6 & 1,6 & 7,5 & 3,2 & 3,5 & 2,7 \\
Rendimiento (m³/hpl/hombre) & 7,1 & 3,0 & 1,9 & 9,2 & 1,2 & 1,5 \\
Rendimiento (m³/hpl/subsistema) & 7,1 & 20,9 & 3,8 & 9,2 & 8,3 & 10,3 \\
Producción por jornada (m/jornada) & 32,7 & 32,4 & 28,7 & 29,8 & 29,4 & 27,7 \\
\hline
\end{tabular}




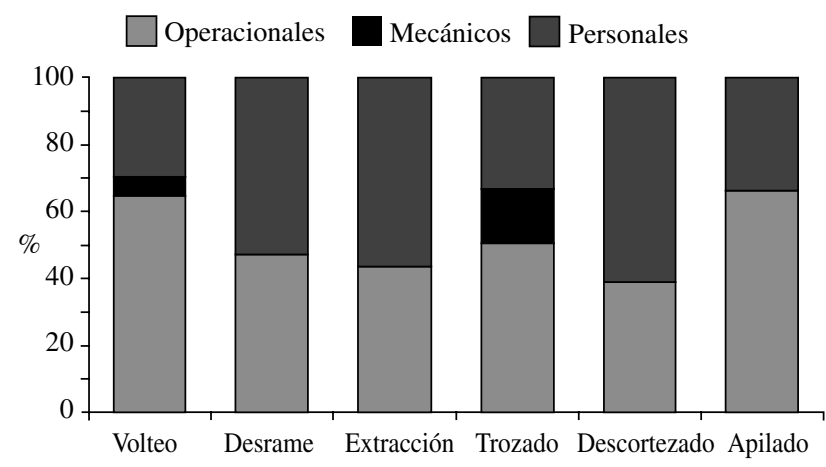

Figura 3. Distribución porcentual de los tiempos no productivos en un sistema de cosecha tradicional de Eucalyptus globulus.

Percent distribution of delays time in a Eucalyptus globulus traditional harvesting system.

origen mecánico, producto de la mantención de la motosierra.

Debido a la configuración del sistema de cosecha, el operador de motosierra no pudo evitar el traslado o desplazamiento hacia la zona de trozado, como tampoco pudo eliminar los tiempos no productivos por mantención mecánica de la motosierra. Sin embargo, el volteo podría aumentar su productividad si se establecieran previamente las pausas de descanso por parte del operador.

En el trozado el operador ocupó gran parte de su tiempo en el desplazamiento hasta la zona de volteo alcanzando un $51 \%$ del tiempo no productivo. Debido a la mayor utilización de la motosierra en la actividad de trozado, la importancia de las demoras mecánicas aumentó en este subsistema (figura 3). Las pausas de descanso del operador ocuparon el 33\% del tiempo no productivo del subsistema trozado.

En el trozado se obtuvo el mejor rendimiento individual de la cuadrilla, con $9,2 \mathrm{~m}^{3} / \mathrm{hpl} / \mathrm{hombre}$. Esto se debió, principalmente, a la necesidad de trozar continuadamente un solo producto de $2,44 \mathrm{~m}$. La ejecución del ciclo de trabajo en el trozado fue muy rápida, alcanzando un promedio de 0,1 minuto por corte.

Los subsistemas desrame, descortezado y apilado presentaron su menor porcentaje de productividad en la actividad de desrame con 50,4\% del tiempo total, mientras que el mayor porcentaje de productividad se presentó en apilado con $76,4 \%$ del tiempo total.

Las pausas por descanso de los trabajadores fueron relevantes en estos subsistemas. De esta forma, las pausas por descanso del desrame ocuparon el 53\% del tiempo no productivo, mientras que el registro de demoras operacionales fue compuesto por la asistencia al trozado (30\% de las demoras) y traslado hacia la zona trozado (17\% de las demoras).

En el descortezado, el $61 \%$ del tiempo no productivo se debió a pausas por descanso. Las demoras operacionales se dividieron en: ordenar los desechos del descortezado (18\% de las demoras), acomodo de la vestimenta $(12 \%)$ y traslado hacia el sector de apilado de trozas $(9 \%)$.

En la actividad de apilado de trozas, los trabajadores presentaron una menor proporción de tiempo de descanso (34\% de las demoras), mientras que en esta actividad, el traslado hacia la zona de volteo pasó a ser el tiempo no productivo de mayor importancia (59\% de las demoras). Por último, la limpieza de las vías de saca concentró el $8 \%$ de los tiempos no productivos.

En cuanto a la productividad de los trabajadores con hachas, en el desrame se obtuvo el mayor rendimiento, con $3 \mathrm{~m}^{3} / \mathrm{hpl} / \mathrm{hombre}$. Toda la cuadrilla en conjunto (siete trabajadores) alcanzó los $20,9 \mathrm{~m}^{3} / \mathrm{hpl}$, destinando en total un promedio de 1,6 horas diarias a esta actividad, de las cuales tan solo el 50,4\% correspondió a tiempo productivo.

\section{DISCUSIÓN}

El sistema de cosecha forestal analizado en plantaciones de Eucalyptus sp. de corta rotación, se caracteriza por la alta ocupación en mano de obra y una baja escala de producción. La cuadrilla consiste en diez trabajadores forestales y dos yuntas de bueyes que obtienen en conjunto una producción promedio de $30,1 \mathrm{~m}^{3} /$ día. Los porcentajes de productividad de los distintos subsistemas fluctúan entre un 40,5\% para el volteo y $76,4 \%$ en apilado de trozas, mientras que los rendimientos por subsistema varían entre $3,8 \mathrm{~m}^{3} / \mathrm{hpl}$ con dos yuntas de bueyes en extracción y $20,9 \mathrm{~m}^{3} / \mathrm{hpl}$ en desrame con siete trabajadores con hachas.

La extracción de la madera realizada principalmente a favor de la pendiente, con distancia de madereo promedio de $50 \mathrm{~m}$, favorece la faena con bueyes (Silverades y Segerström 1983). Sin embargo, el rendimiento individual observado fue bajo al compararlo con otros estudios que presentan rendimientos promedio por sobre los $3 \mathrm{~m}^{3} / \mathrm{h}$ (FAO 1995, Pantaenius 2003). El bajo rendimiento obtenido se explica por las condiciones del sotobosque que dificultan la extracción de trozas, registrando un tiempo promedio de 10,9 minutos en completar un ciclo de extracción, con un promedio de $0,34 \mathrm{~m}^{3}$ por viaje.

La frecuencia y magnitud de los momentos de descanso de los trabajadores es decisiva en la productividad del sistema. Por esto se considera que una mejor organización de estas pausas (preestablecidas) por medio de mayor capacitación de los trabajadores y planificación de la faena, contribuiría a mejorar la productividad del sistema, minimizando la ocurrencia de accidentes, disminuyendo el sobreesfuerzo físico y en el mediano plazo las enfermedades laborales.

En todo caso, frente a mayores requerimientos de producción, se recomienda, para las actividades de 
descortezado y apilado, evaluar la incorporación de mecanización con el propósito de evitar el sobreesfuerzo físico del trabajador y de esta manera aumentar la productividad de la faena.

\section{CONCLUSIONES}

La implementación de un sistema de cosecha forestal tradicional, con utilización intensiva de mano de obra y con herramientas manuales es una opción pertinente en faenas forestales de pequeña escala. Además, es recomendable utilizar este sistema en zonas rurales remotas, en una época del año (otoño, invierno) en la cual existe baja ocupación de mano de obra.

No obstante, para aumentar su productividad, disminuir los riesgos de accidentes y eliminar enfermedades profesionales debe considerarse una mayor organización de los métodos y técnicas de trabajo y, además, se deben establecer las pausas de descanso del personal bien distribuidas durante la jornada.

\section{REFERENCIAS}

Carey P. 1992. Estudio de productividad y rendimiento de un sistema de explotación con skidder. Valdivia, Chile.
Facultad de Ciencias Forestales. 38 p. (Informe de convenio, 206).

Currie R. 1979. Análisis y medición del trabajo. México D.F., México. Diana. 348 p.

Dykstra D, R Heinrich. 1996. Código modelo de prácticas de aprovechamiento forestal de la FAO. Roma. FAO. $85 \mathrm{p}$.

FAO. 1995. Uso de bueyes en operaciones de aprovechamiento forestal en áreas rurales de Costa Rica. Roma. FAO. 41 p. (Estudios monográficos de explotación forestal, 3).

INFOR (Instituto Forestal, CL). 2002. Disponibilidad de madera pulpable de Eucalyptus sp. en Chile período 20012018. Informe Técnico 163. 168 p.

Otavo E. 1984. Extracción de troza mediante bueyes y tractores agrícolas. Roma. FAO. 104 p. (Estudio FAO Montes, 49).

Pantaenius P. 2003. Empleo de bueyes en la extracción de raleo de pino; Ensayo comparativo individual y en yunta en plantaciones de la Patagonia. CIEFAP-Patagonia Forestal. 9(1): 9-12.

Prodan M, R Peters, F Cox, P Real. 1997. Mensura forestal. San José, Costa Rica. IICA. 561 p.

Silverades R, G Segerström. 1983. Tecnología básica en operaciones forestales. Roma. FAO. 122 p. (Estudio FAO Montes, 36). 